\title{
Crecimiento y producción de metabolitos de la cianobacteria marina Synechococcus sp. (Chroococcales) en función de la irradiancia
}

\author{
Néstor Rosales-Loaiza ${ }^{1}$, Miguel Guevara $^{2}$, César Lodeiros $^{2} \&$ Ever Morales $^{1 *}$ \\ 1. Laboratorio de Microorganismos Fotosintéticos, Departamento de Biología, Facultad Experimental de Ciencias, \\ Universidad del Zulia, Maracaibo, Venezuela. Teléfono: +58-261-7597734; Fax: +58-261-7598078; \\ evermster@gmail.com \\ 2. Laboratorio de Acuicultura, Dpto. Biología Pesquera, Instituto Oceanográfico de Venezuela. Cumaná, Venezuela.
}

Recibido 25-I-2007. Corregido 28-IX-2007. Aceptado 29-IV-2008.

\begin{abstract}
Growth and metabolite production of the marine cyanobacterium Synechococcus sp. (Chroococcales) in function to irradiance. Changes in salinity, temperature and irradiance during wet and dry seasons have induced metabolic versatility in cyanobacteria from saline environments. Cyanobacteria from these environments have biotechnological potential for the production of metabolites with pharmaceutical and industrial interest. We studied the growth, dry mass and metabolite production of the cyanobacterium Synechococcus sp. MOF-03 in function of irradiance $\left(78,156\right.$ and $\left.234 \mu \mathrm{mol} \mathrm{q} \mathrm{m} \mathrm{m}^{-2} \mathrm{~s}^{-1}\right)$. All batch cultures were maintained by triplicate in constant aeration, $12: 12 \mathrm{~h}$ photoperiod, $30 \pm 2^{\circ} \mathrm{C}$ and $35 \%$. Maximum values of protein, carbohydrates and lipids, of $530.19 \pm 11.16,408.94 \pm 4.27$ and $56.20 \pm 1.17 \mu \mathrm{g} \mathrm{ml}^{-1}$, respectively, were achieved at 78 $\mu \mathrm{mol} \mathrm{q} \mathrm{m} \mathrm{s}^{-2} \mathrm{~s}^{-1}$. Pigments, analyzed by HPLC, showed maximum values at $78 \mu \mathrm{mol} \mathrm{q} \mathrm{m} \mathrm{s}^{-2}$ for chlorophyll $a$

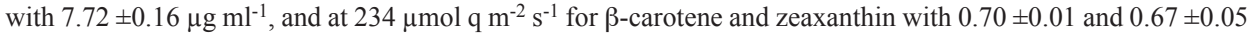

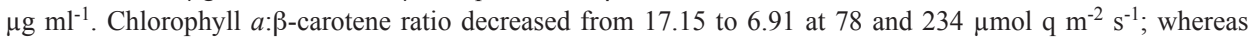

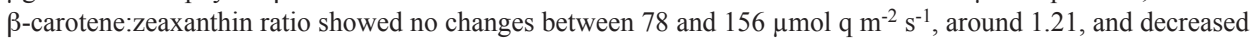
at $234 \mu \mathrm{mol} \mathrm{q} \mathrm{m} \mathrm{s}^{-1}$, to 1.04 . Also, this cyanobacterium produced the greatest cell density and dry mass at $156 \mu \mathrm{mol} \mathrm{q} \mathrm{m}^{-2} \mathrm{~s}^{-1}$, with $406.13 \pm 21.74 \times 10^{6} \mathrm{cell} \mathrm{ml}^{-1}$ and $1.49 \pm 0.11 \mathrm{mg} \mathrm{ml}^{-1}$, respectively. Exopolysaccharide production was stable between 156 y $234 \mu \mathrm{mol} \mathrm{q} \mathrm{m}^{-2} \mathrm{~s}^{-1}$, around $110 \mu \mathrm{g} \mathrm{ml}^{-1}$. This Synechococcus strain shows a great potential for the production of enriched biomass with high commercial value metabolites. Rev. Biol. Trop. 56 (2): 421-429. Epub 2008 June 30.
\end{abstract}

Keywords: Cyanobacteria, biochemical composition, irradiance, Synechococcus.

Las cianobacterias constituyen un diverso grupo de organismos procariotas fotosintéticos que tienen la capacidad de realizar fotosíntesis oxigénica, mediante un mecanismo muy similar al usado por plantas superiores (Garbisu et al. 1999). Además, poseen una gran variedad morfológica, estructural y fisiológica para adaptarse a una amplia gama de parámetros ambientales (Whitton y Potts 2000, Mundt et al. 2001).

Debido a que las cianobacterias son organismos autótrofos, la fuente luminosa es crítica dentro de los factores abióticos que regulan su crecimiento (Becker 1994, Betancourt 1997), y es de gran importancia tomarla en cuenta para el cultivo de cianobacterias a ser utilizadas para la producción de compuestos de valor comercial. Recientemente la fotosíntesis de este grupo de microorganismos ha sido considerada como una vía efectiva para reducir la emisión de dióxido de carbono, un importante gas invernadero, a la atmósfera (Yun y Park 1997). En general, la mayoría de los sistemas de cultivo masivo son limitados por la luz (Yun y Park 2001). Por lo tanto, comprender la dependencia de la luz en la actividad metabólica de microalgas y cianobacterias es de gran importancia para la producción de estos microorganismos 
y de los compuestos de importancia económica que éstos producen durante su crecimiento (Jeon et al. 2005)

Las microalgas y cianobacterias han sido ampliamente estudiadas por varios propósitos, tales como la producción de biomasa como una fuente de compuestos químicos de gran valor económico (Ventosa y Nieto 1995) además de muchos otros usos. Existe un interés creciente por el desarrollo de técnicas eficientes del cultivo de microalgas y cianobacterias, debido a que se confirman como una fuente de sustancias de uso industrial y farmacológico de gran valor económico; tales como clorofila, carotenoides, ficobiliproteínas, proteínas, exopolisacáridos y otros metabolitos biológicamente activos (Paniagua-Michel y Sasson 1995, De Philippis y Vicenzini 1998, Hejazi y Wijffles 2004, García-González et al. 2005, Dayananda et al. 2007). Se han realizados estudios que comprueban el aumento de la producción de pigmentos, proteínas y lípidos a bajas irradiancias (Gordillo et al. 1998). Mientras que la producción de carotenoides y exopolisacáridos mejora notablemente a altas irradiancias (Rücker et al. 1995, Pirog et al. 2002).

Es necesario el estudio de cianobacterias aisladas de ambientes hipersalinos y sometidos a cambios drásticos de las condiciones ambientales en zonas tropicales, debido a que diversas cepas pueden inducir la producción de metabolitos de interés económico cuando son expuestas a condiciones extremas de salinidad, temperatura, irradiancia y deficiencia de nutrientes (Ben-Amozt y Avron 1983).

En este sentido, se ha evaluado el crecimiento y la composición bioquímica de una cepa de Synechococcus aislada de un pozo de agua hipersalino, a diferentes irradiancias en condiciones de laboratorio, bajo la modalidad de cultivos discontinuos.

\section{MATERIALES Y MÉTODOS}

La cianobacteria Synechococcus sp. (Komárek y Anagnostidis 1999) fue aislada del pozo de agua hipersalino Salina Rica $\left(10^{\circ} 47^{\prime} \mathrm{N}\right.$ y $\left.71^{\circ} 38^{\prime} \mathrm{O}\right)$, al norte de la ciudad de
Maracaibo, Venezuela. Este cuerpo de agua es hipersalino, poco profundo y es utilizado para la cosecha de sal en la época seca. Esta cepa de Synechococcus se presenta como células solitarias ovales o cilíndricas, de 3.57 $\pm 0.12 \mu \mathrm{m}$ de longitud y $1.47 \pm 0.09 \mu \mathrm{m}$ de ancho, o formando tricomas de 2 a 4 (y hasta 10) células (Rosales et al. 2005). La cepa se mantiene en la colección permanente del Laboratorio de Microorganismos Fotosintéticos del Departamento de Biología, Facultad Experimental de Ciencias, Universidad del Zulia bajo el código Synechococcus MOF-03.

Los cultivos por triplicado fueron mantenidos en frascos de $350 \mathrm{ml}$ con $150 \mathrm{ml}$ de medio de cultivo, compuesto por agua de mar estéril (salinidad 35\%o) enriquecida con nutrientes (Fábregas et al. 1984) a una concentración de 8 $\mathrm{mM}$ de $\mathrm{NaNO}_{3}$, con una relación N:P de 20:1. Los frascos fueron inoculados con $15 \times 10^{6}$ cel $\mathrm{ml}^{-1}$, mantenidos a $27 \pm 2^{\circ} \mathrm{C}$ con un fotoperiodo luz-oscuridad de 12/12 h. y aireación constante de $5 \mathrm{ml} \mathrm{s}^{-1}$. Se probaron tres diferentes irradiancias, 78,156 y $234 \mu \mathrm{mol}$ quanta $\mathrm{m}^{-2} \mathrm{~s}^{-1}$, mantenidos en cultivos discontinuos hasta alcanzar la fase estacionaria.

La densidad celular fue determinada por recuento en microscopio cada tres días hasta alcanzar fase estacionaria, usando una hematocitómetro Neübauer. La biomasa fue cosechada por centrifugación a $14 \times 10^{3} \mathrm{~g}$ por $15 \mathrm{~min}$. Para los análisis bioquímicos se utilizó biomasa congelada a $-20{ }^{\circ} \mathrm{C}$, excepto para el contenido de pigmentos y peso seco, para los cuales se usó biomasa fresca.

Los pigmentos fueron medidos por HPLC utilizando el método descrito por Vidussi et al. (1996). Su usó una columna Agilent Hypersil MOS (4.6 x $100 \mathrm{~mm}, 5 \mu \mathrm{m}$ de tamaño de partícula), con estándares para la identificación y cuantificación de clorofila $a, \beta$-caroteno y zeaxantina.

Los carbohidratos fueron medidos por el método de fenol-ácido sulfúrico (Kochert 1978) y los exopolisacáridos (EPS) cuantificados por precipitación del sobrenadante con metanol (1:1) (Shah et al. 2000). El contenido de lípidos fue determinado por el método 
de carbonización simple (Marsh y Weinstein 1966); mientras que el de proteínas fue determinado por el método de Lowry et al. (1951) modificado por Herbert et al. (1983). La masa seca fue determinada usando un sistema de filtración Millipore ${ }^{\odot}$ con filtros de fibra de vidrio de $0.45 \mu \mathrm{m}$ mediante el método descrito por Utting (1985).

Los análisis estadísticos se llevaron a cabo con el programa SPSS 10.0 para Windows, utilizando un análisis de varianza de una vía (ANOVA). Cuando se determinaron diferencias significativas entre los tratamientos, se aplicó la prueba Scheffé $(\alpha=0.05)$ de comparaciones múltiples entre medias (Sokal y Rohlf 1995).

\section{RESULTADOS}

El crecimiento de Synechococcus sp. mostró ser una función parabólica en cuanto a la irradiancia; con máximos a $156 \mu \mathrm{mol} \mathrm{q} \mathrm{m}{ }^{-2} \mathrm{~s}^{-1}$ con $406.13 \pm 21.74 \times 10^{6} \mathrm{cel} \mathrm{ml}^{-1} \mathrm{y}$ con diferencia significativa $(\mathrm{p}<0.05)$. La masa seca mostró la misma tendencia observada con la densidad celular, con un máximo a $156 \mu \mathrm{mol} \mathrm{q} \mathrm{m}^{-2} \mathrm{~s}^{-1} \mathrm{de}$ $1.49 \pm 0.11 \mathrm{mg} \mathrm{ml}^{-1}$ (Figura 1).

La Figura 2 muestra el desarrollo de la población durante todo el ensayo, con fases estacionarias cortas de entre seis y nueve días; las cuales son características de esta cepa de cianobacteria. Además también se observó la disminución de la población en los cultivos expuestos a $234 \mu \mathrm{mol} \mathrm{q} \mathrm{m} \mathrm{m}^{-2} \mathrm{~s}^{-1}$ antes que la del resto de los cultivos, evidenciando el efecto inhibitorio de esta alta irradiancia sobre la cianobacteria.

Los cambios en la composición de pigmentos liposolubles de Synechococcus fueron monitoreados por HPLC, obteniendo los cromatogramas con el perfil de dichos pigmentos (Figura 3). La producción de clorofila $a$ fue mayor a la menor irradiancia de $78 \mu \mathrm{mol} \mathrm{q}$ $\mathrm{m}^{-2} \mathrm{~s}^{-1}$ con $7.72 \pm 0.16 \mu \mathrm{g} \mathrm{ml}^{-1}$. Por otro lado, el $\beta$-caroteno y la zeaxantina alcanzaron su máxima producción a la mayor irradiancia de $234 \mu \mathrm{mol} \mathrm{q} \mathrm{m}{ }^{-2} \mathrm{~s}^{-1}$ de $0.70 \pm 0.01$ y $0.67 \pm 0.05$ $\mu \mathrm{g} \mathrm{ml}^{-1}$, respectivamente. Por su parte, la relación clorofila a/ $\beta$-caroteno disminuyó con el

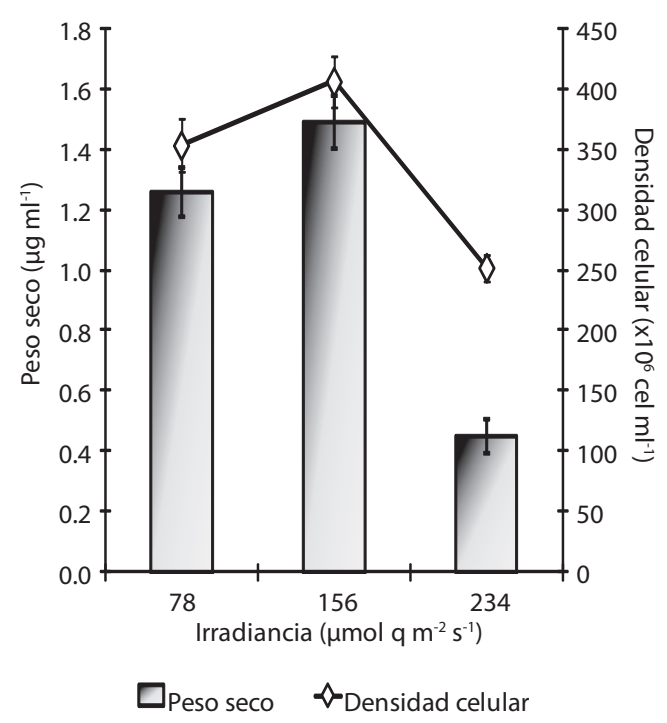

Fig. 1. Densidad celular $\left(\times 10^{6} \mathrm{cel} \mathrm{ml}^{-1}\right)$ y peso seco $(\mu \mathrm{g}$ $\left.\mathrm{cel}^{-1}\right)$ de Synechococcus sp. en relación a la irradiancia

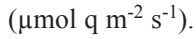

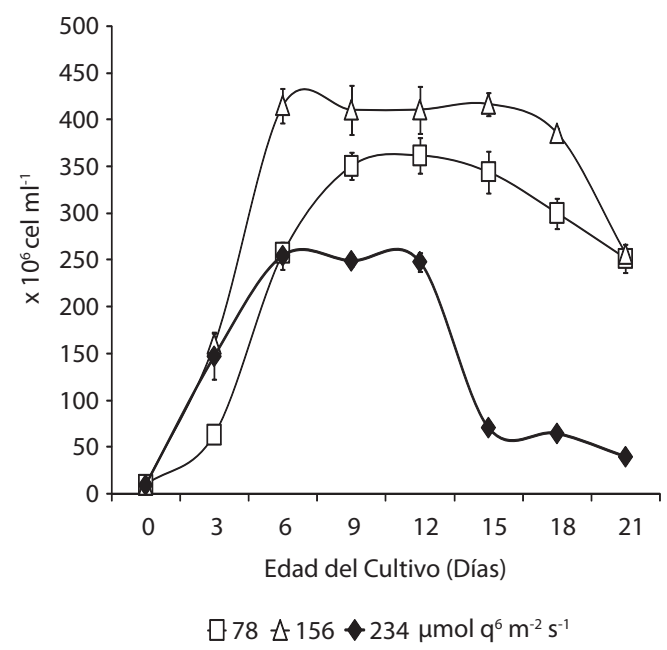

Fig. 2. Curva de crecimiento de Synechococcus sp. en rel-

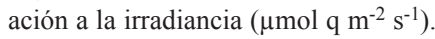

aumento de la irradiancia de 17.15 hasta 6.91 a 78 y $234 \mu \mathrm{mol} \mathrm{q} \mathrm{m}{ }^{-2} \mathrm{~s}^{-1}$, respectivamente y con diferencias significativas $(\mathrm{p}<0.05)$. Mientras que la relación $\mathbf{\square}$-caroteno/ zeaxantina se mantuvo estable a irradiancias bajas e intermedias con cerca de 1.21, disminuyendo a la mayor 


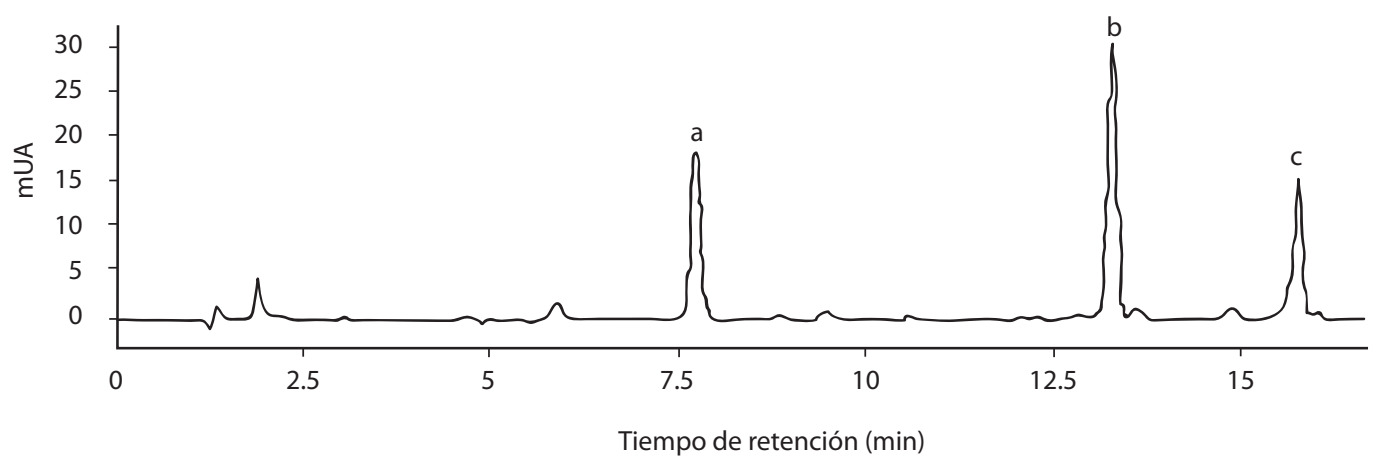

Fig. 3. Cromatograma de HPLC mostrando el perfil de pigmentos de Synechococcus sp. Picos marcados: (a) zeaxantina, (b) clorofila $a$ y (c) $\beta$-caroteno.

irradiancia de $234 \mu \mathrm{mol} \mathrm{q} \mathrm{m} \mathrm{m}^{-2} \mathrm{~s}^{-1}$ hasta 1.04 (Cuadro 1).

La producción de proteínas y lípidos mostró la misma tendencia observada con la clorofila, disminuyendo con la irradiancia con máximos a $78 \mu \mathrm{mol} \mathrm{q} \mathrm{m}^{-2} \mathrm{~s}^{-1}$ de $530.19 \pm 11.16$ y $56.20 \pm 1.17 \mu \mathrm{g} \mathrm{ml}^{-1}$ (Figura 4), representando una diferencia de dos y siete veces respectivamente con respecto a la mayor irradiancia, todos con diferencia significativa $(\mathrm{p}<0.05)$.

La producción de carbohidratos y EPS mostraron tendencias distintas. Por un lado la de carbohidratos alcanzó los máximos valores a la menor irradiancia de $78 \mu \mathrm{mol} \mathrm{q} \mathrm{m} \mathrm{m}^{-2} \mathrm{~s}^{-1}$ con $408.94 \pm 4.27 \mu \mathrm{g} \mathrm{ml}^{-1}$ (Figura 5). Por su parte, la producción EPS parece estar en función de la intensidad luminosa, estabilizándose a irradiancias entre 156 y $234 \mu \mathrm{mol} \mathrm{q} \mathrm{m}{ }^{-2} \mathrm{~s}^{-1}$, con

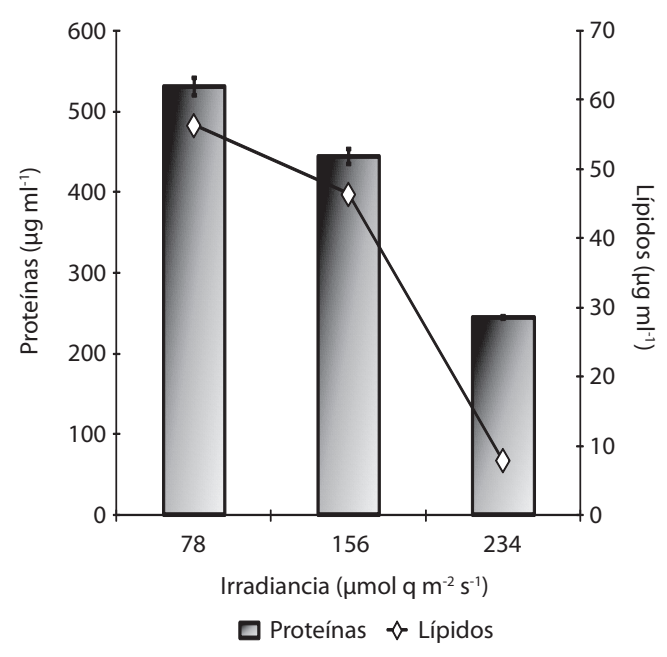

Fig. 4. Producción de proteínas y lípidos de Synechococcus sp. en relación a la irradiancia.

\section{CUADRO 1}

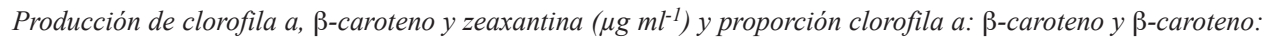
zeaxantina de Synechococcus sp. en relación a la irradiancia ( $\mu \mathrm{mol} \mathrm{q} \mathrm{m}^{-2} \mathrm{~s}^{-1}$ ).

$\begin{array}{lc}\text { Clorofila } a & 7.72 \pm 0.16 \\ \beta \text {-caroteno } & 0.45 \pm 0.03 \\ \text { Zeaxantina } & 0.37 \pm 0.03 \\ \text { Clo } a / \beta \text {-car } & 17.15 \\ \beta \text {-car } / \text { Zeaxan } & 1.21\end{array}$

$7.36 \pm 0.09$
$0.55 \pm 0.02$
$0.45 \pm 0.03$
13.38
1.22

$4.84 \pm 0.18$

$0.70 \pm 0.01$

$0.67 \pm 0.05$

6.91

1.04 


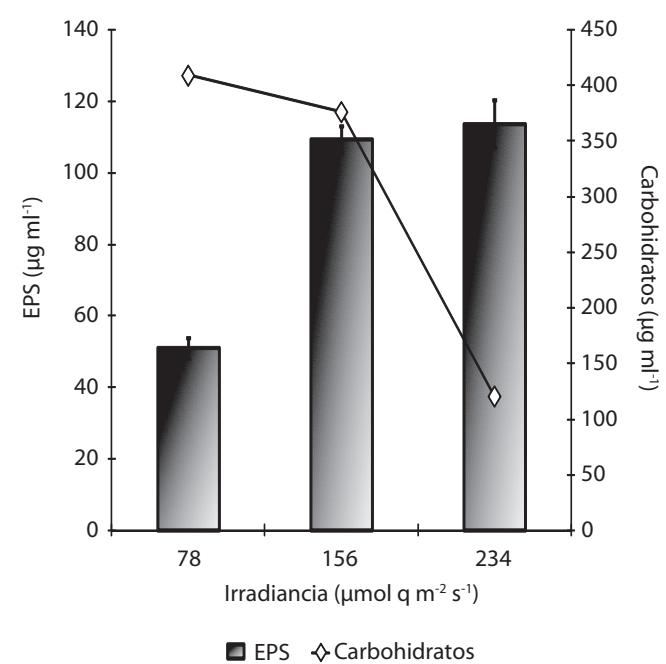

Fig. 5. Producción de carbohidratos y exopolisacáridos $\left(\mu \mathrm{g} \mathrm{ml}^{-1}\right.$ ) de Synechococcus sp. en relación a la irradiancia

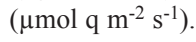

valores de $109.33 \pm 3.79$ y $113.78 \pm 6.63 \mu \mathrm{g}$ $\mathrm{ml}^{-1}$, respectivamente y sin diferencias significativas ( $p>0.05$ ); siendo estos valores el doble con respecto a los alcanzados a la más baja irradiancia (Figura 5).

\section{DISCUSIÓN}

La cianobacteria Synechococcus sp. muestra una preferencia a crecer a bajas e intermedias irradiancias. Este comportamiento ha sido descrito en poblaciones fitoplanctónicas, incluyendo a cepas de Synechococcus, las cuales se sitúan en la base de la zona eufótica (Mastala et al. 1996). Se han realizado diversos trabajos sobre la influencia de la iluminación en cianobacterias y muchas de ellas se caracterizan por crecer de manera óptima a irradiancias bajas e intermedias, como por ejemplo Spirulina platensis (Baldia et al. 1991), Pseudanabaena galeata (Romo 1994), Anabaena PCC7120 (Loreto et al. 2003) y Anabaena variabilis (Yoon et al. 2007). Dentro de esta lista se incluyen también otras cepas de Synechococcus sp., consideradas de forma general como extremadamente tolerante a altas irradiancias (Beljanin y Trenkenšu 1977).
El aumento de la producción de clorofila $a$ a bajas irradiancias; tal y como se observó en los cultivos de esta cianobacteria; es un hecho bastante conocido y reportado en otras cianobacterias como Anabaena (MartínTrillo 1995), Spirulina subsalsa (Tomaselli y Margheri 1995), Plectonema boryanum UTEX 485 (Miśkiewicz et al. 2000) y Anabaena PCC7120 (Loreto et al. 2003) y se produce básicamente por un efecto de ensombrecimiento. Las células al encontrarse en un medio con poca iluminación aumentan la cantidad de membranas tilacoides y de ficobilisomas para captar la mayor cantidad de energía radiante posible (Tandeau de Marsac y Houmard 1993). Así al aumentar la irradiancia, la cantidad de estas estructuras necesarias será menor, debido a la mayor cantidad de energía radiante disponible.

El aumento del contenido de los pigmentos accesorios como $\beta$-caroteno y zeaxantina ha sido observado en cianobacterias carotenogénicas como Planktothrix agardhii (Rücker et al. 1995) y Plectonema boryanum UTEX 485 (Miśkiewicz et al. 2000), en las cuales se produce una inducción de la síntesis de carotenoides en respuesta a elevadas irradiancias y evitar así la fotooxidación (Nigoyi et al. 1997).

La relación clorofila $a / \beta$-caroteno, muestra ese aumento de concentración de clorofila $a$ hallado a bajas irradiancias siendo el valor 2.5 veces mayor que el hallado a la mayor irradiancia. Por su parte, la relación $\beta$-caroteno/ zeaxantina se mantuvo estable a irradiancias bajas e intermedias, disminuyendo ligeramente a la mayor de las irradiancias probadas.

A pesar de esto, el aumento de los carotenoides fue muy leve (aunque estadísticamente significativo, $\mathrm{p}<0.05$ ) y posiblemente, esta cianobacteria activa otro proceso de aclimatación o de control para evitar la fotooxidación de los pigmentos a elevadas intensidades luminosas; tal como se ha descrito en las cianobacterias Synechococcus PCC 7002, Synechococcus PCC 6301 y Microcystis aeruginosa; en las cuales no hay variación en los contenidos de carotenoides (Tandeau de Marsac y Houmard 1993). 
En un estudio realizado por Schagerl y Müller (2006) con cuatro cepas de cianobacterias filamentosas, hallaron que al comparar los tratamientos de baja y alta irradiancia, las bajas irradiancias causaron aumento del contenido de clorofila $a$, observando además un significante aumento de la relación chl a/carotenoides bajo estas mismas condiciones en Nostoc sp. y Anabaena cilindrica. Por otro lado hallaron un aumento de los carotenoides en Anabaena torulosa y Anabaenopsis elenkinii, indicando su acumulación celular. Esto contrasta con algunos trabajos, como el de Millie et al. (1990), donde se reporta que la abundancia relativa de carotenoides, pero no su contenido total, varía con la irradiancia.

Los resultados muestran que esta cepa de Synechococcus posee óptimos de irradiancia distintos para el crecimiento y producción de pigmentos. Es decir, irradiancia a $156 \mu \mathrm{mol} \mathrm{q}$ $\mathrm{m}^{-2} \mathrm{~s}^{-1}$ estimula su crecimiento y a $78 \mu \mathrm{mol} \mathrm{q}$ $\mathrm{m}^{-2} \mathrm{~s}^{-1}$ favorecen la síntesis de clorofila $a$. Esto demuestra que la manipulación de los cultivos para la producción de biomasa enriquecida con pigmentos debe estar en función de la iluminación tomando en cuenta la calidad de luz, irradiancia y fotoperíodo.

En otras cianobacterias y microalgas se ha reportado cambios en el contenido proteico con la intensidad luminosa mejorando la producción a irradiancias bajas e intermedias, lo cual está estrechamente relacionado con la capacidad de asimilación de nitrato inorgánico (Ben-Amotz 1987, Loreto et al. 2003), debido a que este proceso es dependiente de la fotofosforilación y de la irradiancia (Tischner y Lorenzen 1979). Además, a parte de la clorofila y los carotenoides, las ficobiliproteínas son otro grupo importante de pigmentos presentes en cianobacterias $\mathrm{y}$, en muchos casos, representan la mayor parte de los pigmentos accesorios dentro de la célula; constituyendo un gran fracción del contenido proteico total (Boussiba y Richmond 1980).

En Spirulina y Oscillatoria spp., se ha descrito que la incorporación relativa de proteínas a las células es inversamente proporcional al incremento de la intensidad luminosa.
Posiblemente, a altas irradiancias se induce una elevada tasa de asimilación de carbono total, de tal manera que la incorporación de carbono a la fracción proteica, es menor con respecto a la obtenida en la fracción de polisacáridos bajo estas condiciones (van Rijn y Shilo 1986).

La disminución del contenido lipídico con el aumento de la irradiancia ha sido reportada en otros microorganismos fotosintéticos. Tal efecto, parece estar relacionado con el efecto de ensombrecimiento y la consecuente disminución de los constituyentes de las membranas tilacoidales (Gordillo et al. 1998). Más específicamente, esto parece estar relacionado con la disminución de los galactolípidos enriquecidos con ácido eicosapentanoico, constituyentes de dichas membranas. De tal manera que a una mayor disponibilidad de luz, se necesitan menos componentes estructurales funcionales para la captación de la energía radiante (Maseda 2002).

Las condiciones óptimas para la producción de EPS no coinciden con las óptimas para el crecimiento. No sólo en esta cepa de Synechococcus, sino también en otras cianobacterias como Anabaena sp. ATCC 33047 (Moreno et al. 1998). La producción mejora en condiciones extremas de limitación (o exceso) de nutrientes e irradiancia; con altos valores a altas intensidades lumínicas (Giroldo y Vieira 2002).

La producción de metabolitos de interés económico en cultivos de la cianobacteria marina Synechococcus sp. en cultivos está regulada por la irradiancia aplicada a dichos cultivos, obteniéndose una buena producción de biomasa enriquecida a intensidades lumínicas intermedias. Para la producción de clorofila, proteínas y lípidos es aumentada a bajas e intermedias irradiancias; mientras que la producción de carotenoides y exopolisacáridos tiene mejores rendimientos a altas irradiancias.

\section{AGRADECIMIENTOS}

Este trabajo ha sido financiado por FONACIT-Venezuela, a través del proyecto \# S1-2000000786. 


\section{RESUMEN}

Las cianobacterias de ambientes salinos presentan una versatilidad metabólica inducida por los cambios de salinidad, temperatura e irradiancia, durante los períodos de sequía y lluvias. Por ello es importante la búsqueda en estos ambientes de cianobacterias con potencial biotecnológico para la producción de metabolitos de interés farmacéutico e industrial. Se reporta el crecimiento, masa seca y producción de metabolitos de la cianobacteria Synechococcus sp. MOF-03 en función de la irradiancia (78, 156 y $234 \mu \mathrm{mol}$ $\left.\mathrm{q} \mathrm{m}^{-2} \mathrm{~s}^{-1}\right)$. Los cultivos discontinuos por triplicado, fueron mantenidos con aireación constante, fotoperiodo 12:12 h, $30 \pm 2^{\circ} \mathrm{C}$ y a $35 \%$. Los máximos valores de proteínas, carbohidratos y lípidos de $530.19 \pm 11.16,408.94 \pm 4.27$ y $56.20 \pm 1.17 \mu \mathrm{g} \mathrm{ml}^{-1}$ respectivamente, fueron obtenidos a $78 \mu \mathrm{mol} \mathrm{q} \mathrm{m} \mathrm{m}^{-2} \mathrm{~s}^{-1}$. Los pigmentos, analizados por HPLC, mostraron los máximos a $78 \mu \mathrm{mol} \mathrm{q} \mathrm{m}{ }^{-2} \mathrm{~s}^{-1}$ para clorofila $a$ con $7.72 \pm 0.16 \mu \mathrm{g} \mathrm{ml}^{-1}$; y a $234 \mu \mathrm{mol} \mathrm{q} \mathrm{m} \mathrm{m}^{-2} \mathrm{~s}^{-1}$ para $\beta$-caroteno y zeaxantina con $0.70 \pm 0.01$ and $0.67 \pm 0.05$ $\mu \mathrm{g} \mathrm{ml}^{-1}$. La relación clorofila $a$ : $\beta$-caroteno disminuyó de 17.15 hasta 6.91 a 78 y $234 \mu \mathrm{mol} \mathrm{q} \mathrm{m}{ }^{-2} \mathrm{~s}^{-1}$; mientras que la relación $\beta$-caroteno:zeaxantina se mantuvo sin cambios entre 78 y $156 \mu \mathrm{mol} \mathrm{q} \mathrm{m}{ }^{-2} \mathrm{~s}^{-1}$, con cerca de 1.21 y disminuyó a $234 \mu \mathrm{mol} \mathrm{q} \mathrm{m}{ }^{-2} \mathrm{~s}^{-1}$ a 1.04 . La cianobacteria produjo la mayor densidad celular y masa seca a $156 \mu \mathrm{mol} \mathrm{q} \mathrm{m}$ $\mathrm{s}^{-1}$, con $406.13 \pm 21.74 \times 10^{6} \mathrm{cel} \mathrm{ml}^{-1}$ y $1.49 \pm 0.11 \mathrm{mg} \mathrm{ml}^{-1}$ respectivamente. La producción de exopolisacáridos se mantuvo alrededor de $110 \mu \mathrm{g} \mathrm{ml}^{-1}$ entre 156 y $234 \mu \mathrm{mol} \mathrm{q}$ $\mathrm{m}^{-2} \mathrm{~s}^{-1}$. Así, esta cepa de Synechococcus muestra un gran potencial para la producción de biomasa enriquecida con metabolitos de alto valor comercial.

Palabras clave: Cianobacteria, composición bioquímica, irradiancia, Synechococcus.

\section{REFERENCIAS}

Baldía, S., T. Nishijima \& Y. Hata. 1991. Effects of physico-chemical factors and nutrients on the growth of Spirulina platensis isolated from Lake Kojima, Japan. Suisan Gakkaishi Bull. Jap. Soc. Sci. 57: 481-490.

Becker, E. 1994. Microalgal Biotechnology and Microbiology, Cambridge University, Cambridge, Reino Unido.

Beljanin, V. \& A. Trenkenšu. 1977. Growth and spectrophotometric characteristics of the blue-green alga Synechococcus elongatus under different temperature and light conditions. Arch. Hydrobiol. 51, Algol. Studies 18: 46-66.
Ben-Amotz, A. \& M. Avron. 1983. Accumulation of metabolites by halotolerant algae and its industrial potential. Ann. Rev. Microbiol. 37: 95-119.

Ben-Amotz A. 1987. Effect of irradiance and nutrient deficiency on the chemical composition of Dunaliella bardawil Ben-Amotz \& Avron (Volvocales, Chlorophyta). J. Plant Physiol. 1131: 479-487.

Betancourt L. 1997. Producción, purificación y caracterización de la ficocianina de Synechococcus sp. IO-9201 aislada en aguas de Cuba. Tesis de Doctorado, Universidad de La Coruña, España.

Borowitzka, M. 1995. Microalgae as a source of pharmaceuticals and other biologically active compounds. J. Appl. Phycol. 7: 3-15.

Boussiba, S. \& A. Richmond. 1980. C-phycocianin as storage protein in the blue-green algae Spirulina platensis. Arch. Microbiol. 125: 143-147.

Dayanandaa, C., R. Saradaa, M. Usha Ranib, T. Shamalab \& G. Ravishankara. 2007. Autotrophic cultivation of Botryococcus braunii for the production of hydrocarbons and exopolysaccharides in various media. Biomass Bioenergy 31: 87-93.

De Philippis, R. \& M. Vincenzini. 1998. Exocellular polysaccharides from cyanobacteria and their possible applications. FEMS Microbiol. Rev. 22: 151-175.

Fábregas, J., J. Abalde, C. Herrero, B. Cabezas \& M. Veiga. 1984. Growth of marine microalgae Tetraselmis suecica in batch cultures with different salinities and nutrient concentration. Aquaculture 51: 237-243.

Garbisu, C., A. Blanco, I. Alkorta, M. Llama \& J. Serra. 1999. Biotecnología con cianobacterias. Investigación Ciencia 65-71.

García-González, M., J. Moreno, J. C. Manzano, F. Florencio \& M. Guerrero. 2005. Production of Dunaliella salina biomass rich in 9-cis- $\beta$-carotene and lutein in a closed tubular photobioreactor. J. Biotechnol. 115: 81-90.

Giroldo, D. \& A. Vieira. 2002. An extracellular sulfated fucose-rich polysaccharide produced by a tropical strain Cryptomonas obovata (Cryptophyceae). J. Appl. Phycol. 14: 185-191.

Gordillo, F., M. Goutx, F. Figueroa \& F. Niell. 1998. Effects of light intensity, $\mathrm{CO}_{2}$ and nitrogen supply on lipid class composition of Dunaliella viridis. J. Appl. Phycol. 10: 135-144. 
Hejazi, M. \& R. Wijffels. 2004. Milking microalgae. Trends Biotechnol. 22: 189-194.

Herbert, D., P. Phipps \& P. Stronoe. 1971. Chemical analysis of microbial cells, pp. 209-344. In J. Norris \& D. Ribbons (eds.). Methods in Microbiology. Academic, Londres, Reino Unido.

Jeon, Y., C. Cho \& Y. Yun. 2005. Measurement of microalgal photosynthetic activity depending on light intensity and quality. Biochem. Engin. J. 27: 127-131.

Kochert, G. 1978. Carbohydrate determination by the phenol-sulfuric acid method, pp. 95-97. In J. Hellebust \& J. Craigie (eds.). Handbook of Phycological Methods. Physiological and Biochemical Methods. Cambridge University, Cambridge, Reino Unido.

Komárek, J. \& K. Anagnostidis. 1999. Cyanoprokaryota: 1. Teil Chroococcales. In: H. Ettl, G. Gärtner, H. Heynig and D. Mollenhauer (eds.), Sübwasserflora von Mitteleuropa. Gustav Fisher, Jenna, Alemania.

Loreto, C., N. Rosales, J. Bermúdez \& E. Morales. 2003 Producción de pigmentos y proteínas de la cianobacteria Anabaena PCC 7120 en relación a la concentración de nitrógeno e irradiancia. Gayana Botánica 60: 83-89.

Lowry, O., H. Rosenbrough, A. Farr \& R. Randall. 1951. Protein measurement with the folin-phenol reagent. J. Biol. Biochem. 193: 265-275.

Marsh, J. \& D. Weinstein. 1966. Simple charring method for determination of lipids. J. Lipid Res. 7: 574-576.

Martín-Trillo, M. 1995. Afloramientos masivos (Blooms) de cianobacterias en los arrozales valencianos: Seguimiento de su desarrollo y caracterización de dos estirpes formadoras. Tesis de Licenciatura. Universidad Autónoma de Madrid, España.

Maseda, A. 2002. La luz modifica la productividad, la composición y el proteoma de microalgas del género Nannochloropsis. Tesis de Doctorado. Universidad de Santiago de Compostela, España.

Mastala, Z., S. Herodek, K. Balogh, G. Borbely, H Shafik \& L. Voeroes. 1996. Nutrient requirement and growth of a Synechococcus species isolated from Lake Balaton. Inter. Rev. Gesamt. Hydrobiol. 81: 503-512.

Miśkiewicz, E., A. Ivanov, J. Williams, M. Khan, S. Falk $\&$ N. Huner. 2000. Photosynthetic acclimation of the filamentous cyanobacterium Plectonema boryanum UTEX 485, to temperature and light. Plant Cell Physiol. 41: 767-775.
Moreno, J., M. Vargas, H. Olivares, J. Rivas \& M. Guerrero. 1998. Exopolysaccharide production by the cyanobacterium Anabaena sp. ATCC 33047 in batch and continuous culture. J. Biotechnol. 60: 175-182.

Mundt, S., S. Kreitlow, A. Nowotny \& U. Effmert. 2001. Biochemical and pharmacological investigations of selected cyanobacteria. Int. J. Hyg. Environ. Health 203: 327-334.

Nigoyi, K., O. Björkman \& R. Grossman. 1997. The roles of specific xanthophylls in photoprotection. Proc. Natl. Acad. Sci. 94: 14162-14167.

Paniagua-Michel, J. \& A. Sasson. 1995. Moléculas de microalgas de importancia económica, pp. 297-310. In K. Alveal, M. Ferrario, E. Oliveira \& E. Sar (eds.). Manual de Métodos Ficológicos. Concepción, Chile.

Pirog, T., T. Grinberg \& Y. Malashenko. 2002. Strategy of obtaining microbial exopolisaccharides possessing stable preset proprieties. Microbiol. 64: 81-94.

Romo, S. 1994. Growth parameters of Pseudanabaena galeata Böcher in culture under different light and temperature conditions. Algol. Studies 75: 239-248.

Rosales, N., J. Ortega, R. Mora \& E. Morales. 2005. La salinidad como factor modulador del crecimiento y composición bioquímica de la cianobacteria Synechococcus sp. Ciencias Marinas 31: 349-355.

Rücker, J., J. Kohl \& K. Kaiser. 1995. Response of carotenoids to variations of growth-limiting factors in three filamentous blue-green algae. Algol. Studies 77: 51-65.

Shah, V., A. Ray, N. Garg \& D. Madamwar. 2000 Characterization of the extracellular polysaccharide produced by a marine cyanobacterium Cyanothece sp. ATCC 51142, and its exploitation toward metal removal from solutions. Current Microbiol. 40: 274-278.

Sokal, R. \& F. Rohlf. 1995. The principles and practice of statistics in biological research. W. H. Freeman, Nueva York, Estados Unidos.

Tandeau De Marsac, N. \& J. Houmard. 1993. Adaptation of cyanobacteria to environmental stimuli: new steps towards molecular mechanisms. FEMS Microbiol. Rev. 104: 119-190.

Tischner, R. \& H. Lorenzen. 1979. Nitrate uptake and nitrate reduction in synchronous Chlorella. Planta 146: 287. 
Tomaselli, L. \& M. Margheri. 1995. Effects of light on pigments and photosynthetic activity in a phycoerithrinrich strain of Spirulina subsalsa. Aquat. Microbial Ecol. 9: 27-31.

Utting, S. 1985. Influence of nitrogen availability on the biochemical composition of three unicellular marine algae of commercial importance. Aquacul. Engin. 4: 175-190.

van Rijn, J. \& M. Shilo. 1986. Nitrogen limitation in natural populations of cyanobacteria (Spirulina and Oscillatoria spp.) and its effect on macromolecular synthesis. Appl. Environ. Microbiol. 52: 340-344.

Ventosa, A. \& J. Nieto. 1995. Biotechnological applications and potentialities of halophilic microorganism. World J. Microbiol. Biotechnol. 11: 85-94.

Vidussi, F., H. Claustré, J. Bustillos, C. Cailliau \& J. Marty. 1996. Determination of chlorophylls and carotenoids of marine phytoplankton: separation of chlorophylls $a$ from divinyl-chlorophyll $a$ and zeaxanthin from lutein. J. Plank. Res. 18: 237-282.

Whitton B. \& M. Potts. 2000. Introduction to cyanobacteria, p. 1-11. In B. Whitton \& M. Potts (eds.). The ecology of cyanobacteria. Kluwer Academic, Dordrecht, Países Bajos. pp. 1-19.

Yoon, J., J. Shin \& T. Park. 2007. Characterization of factors influencing the growth of Anabaena variabilis in a bubble column reactor. Biores. Technol. En prensa (disponible en-línea en www.sciencedirect.com)

Yun, Y. S., J.M. Park. 1997. Development of gas recycling photobioreactor system for microalgal carbon dioxide fixation. Korean J. Chem. Eng. 14: 1297-1300.

Yun, Y. S., J.M. Park. 2001. Attenuation of monochromatic and polychromatic lights in Chlorella vulgaris suspensions. Appl. Microbiol. Biotechnol. 55: 765-770. 